\title{
Fault-tolerant Control Design to Enhance Damping of Inter-area Oscillations in Power Grids
}

\author{
F. R. Segundo Sevilla ${ }^{1 * \dagger}$, I. Jaimoukha ${ }^{1}$, B. Chaudhuri ${ }^{1}$ and P. Korba ${ }^{2}$ \\ ${ }^{1}$ Department of Electrical and Electronic Engineering, Imperial College London, London, UK \\ 2 Zurich University of Applied Sciences, Winterthur, Switzerland
}

\begin{abstract}
SUMMARY
In this paper passive and active approaches for the design of fault-tolerant controllers (FTCs) are presented. The FTCs are used to improve the damping of inter-area oscillations in a power grid. The effectiveness of using a combination of local and remote (wide-area) feedback signals is first demonstrated. The challenge is then to guarantee a minimum level of dynamic performance following a loss of remote signals. The designs are based on regional pole-placement using Linear Matrix Inequalities (LMIs). First, a passive FTC is proposed. It is shown that the computation of the controller reduces to the solution of bilinear matrix inequalities. An iterative procedure is then used to design the controller. Next, as an alternative to active, time varying controllers, one for each fault scenario, we propose an approach for the design of a 'minimal switching' FTC in which only one controller is designed, but where a simple switch is incorporated into the controller structure. A case study in a linear and nonlinear Nordic equivalent system is presented to show that the closed-loop response using a conventional control (CC) design could deteriorate the performance or even destabilize the system if the remote signals are lost and to demonstrate the effectiveness of the proposed FTC designs.
\end{abstract}

KEY WORDS: Fault-tolerant control, regional pole-placement, simultaneous design, power oscillation damping, local and remote feedback.

\section{INTRODUCTION}

With the increase in the requirements of regulation and operation of control systems, the topic of fault-tolerant control (FTC) has attracted the interest of research works in different areas (e.g., $[1,2,3,4])$. In general, repair and maintenance services cannot be provided quickly, making FTC an important design strategy. The objective of FTC is to design an appropriate controller such that the resulting closed-loop system can tolerate abnormal operations of specific control components and retain overall system stability with acceptable system performance [5]. Within FTC theory, several approaches have been reported: the algebraic Riccati equation-based approach [6], the coprime factorization approach [7], the HamiltonJacobi-based approach [8], the sliding-mode control approach [9] and the LMI approach [10]. Among the mentioned studies, the LMI approach is relatively simpler to be implemented, making this approach one of the most popular in the field; see $[11,12]$ and the references therein.

In FTC theory, two different approaches exist in the literature: active and passive FTCs. In active schemes, the controller is reconfigured whenever a fault is detected. In passive FTC schemes, the controller is fixed; the fault tolerance is obtained by an a priori design based on the fault models, such that the controller is able to handle all possible faults [13]. Niemann and Stoustrup [14, 15, 13] have demonstrated the effectiveness of passive schemes and have also pointed out the benefits and

${ }^{*}$ Correspondence to: Segundo Sevilla Felix Rafael, Department of Electrical and Electronic Engineering, Imperial College London, South Kensigton Campus, SW7 2AZ, London, UK.

†Email: frs09@ic.ac.uk 
drawbacks of using both schemes. Since the power industry is used to passive control structures, in this work we concentrate on this particular scheme, although we also propose an active scheme which does not require a full reconfiguration of the controller.

In the field of power grids, cascading failures in several power systems worldwide have attracted the interest of many researchers. These events normally occur due to the loss of generating units, breaking failures, circuit outages or a combination of system conditions and events (requiring robust control design and, by consequence, providing the opportunity for using FTC schemes) [16]. With the state-of-the-art wide-area measurement systems (WAMS) infrastructure, power oscillation damping using remote (or wide-area) signals is certainly feasible. This could potentially improve the stability limits and allow operation of the transmission lines closer to their thermal capacity, without compromising security. Utilities however, are concerned about the consequences of unacceptable delay or complete loss of one or more of the remote feedback signals which could jeopardize the dynamic performance of their system.

With the introduction of phasor measurement units (PMUs), the monitoring and analysis of power systems dynamics have been significantly improved $[17,18,19]$. PMUs provide measurements of important quantities such as voltages, currents and active and reactive powers from different remote locations in the power system. Although the measurements from the PMUs are not likely to fail, the effects of a failure could be severe; this implies open research opportunities such as the fault-tolerant control area. Conventional control (CC) schemes may be unable to work in critical scenarios. FTCs increase the reliability and maintain the stability of the system even if a remote signal is lost.

Several techniques have been reported in the literature to tackle the adverse impact of latency or delay involved in communicating the remote signals [20,21, 22]. Another potential problem could be low data rate/bandwidth availability which is likely to be encountered for networked communication if WAMS infrastructure were to be shared between multiple data intensive services in the future. A solution to this problem was presented in [23]. Despite a number of papers on latency and less so on the bandwidth problem, very little has been reported on tackling the situation where one or more remote signals are completely lost. The impact of loss of signals on the stability of inter-connected power systems is shown in [24] but no technique is presented to resolve the problem.

In this work, a case study on the Nordic equivalent system [25] with two poorly damped interarea modes is presented. Supplementary power oscillation damping (POD) control of a static var compensator (SVC) [26] installed near Oslo is considered for improving the damping of the two inter-area modes. It is shown that using only local signals, it is not possible to achieve the specified target of $10 \%$ damping ratio which justifies the use of remote signals. Using a combination of a local and a remote signal, a conventional controller is shown to produce the desired performance under normal condition (i.e. when the remote signal is present). However, in case of loss of the remote signal, the system response deteriorates as in the open-loop response (no POD on SVC) which is not acceptable. Also, a linear simulation is presented to show how the $\mathrm{CC}$ design can affect the stability of the closed-loop system if remote signals are used and one of them is lost.

A passive fault-tolerant control design methodology is then proposed to ensure an acceptable performance level even in case of loss of remote signals. The FTC design methodology is based on simultaneous regional pole-placement for multiple operating conditions, i.e. normal (remote signals present) and loss of (remote) signals, and the algorithm to calculate the controller is described. A special architecture for an active FTC design is next proposed. This 'minimal switching' architecture combines the attractiveness of the passive approach (simple implementation) and the active approach (incorporating fault information). The effectiveness of both types of FTC designs against the $\mathrm{CC}$ is then demonstrated using the Nordic equivalent system.

\section{CONTROL DESIGN FORMULATION}

Consider the following state space representation of a linear time-invariant (LTI) system

$$
\dot{x}(t)=A x(t)+B u(t), \quad y(t)=C x(t), \quad G(s) \stackrel{s}{=}\left[\begin{array}{c|c}
A & B \\
\hline C & 0
\end{array}\right]
$$




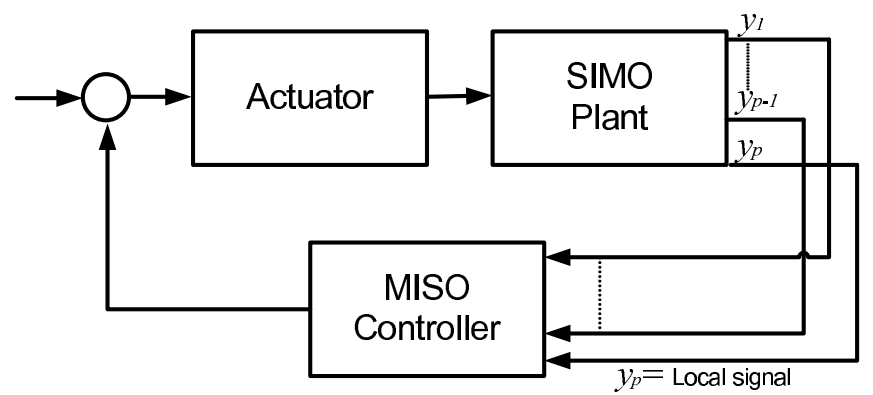

Figure 1. General control formulation: Wide-area signals subject to faults

where $x \in \Re^{n}, u \in \Re^{q}$ and $y \in \Re^{p}$ are the state, input and output vectors, respectively. $A \in \Re^{n \times n}$, $B \in \Re^{n \times q}$ and $C \in \Re^{p \times n}$ are the state, input and output distribution matrices of the system, respectively. The output matrix $C$ is described as

$$
C=\left[\begin{array}{lllll}
c_{1}^{T} & c_{2}^{T} & \ldots & c_{p-1}^{T} & c_{p}^{T}
\end{array}\right]^{T}
$$

where $c_{j} \in \Re^{1 \times p}$ represents the $j$ th output of the system. In this work $p \geq 2$, one local and at least one remote signal are used. The general concept is described in Figure 1. The sensor faults (or loss of signals) can be represented by a family of plants

$$
\dot{x}(t)=A x(t)+B u(t), \quad y_{i}(t)=C_{i} x(t), \quad G_{i}(s) \stackrel{s}{=}\left[\begin{array}{c|c}
A & B \\
\hline C_{i} & 0
\end{array}\right]
$$

where, for $i=0,1, \ldots, p-1$ and $j=1,2, \ldots, p-1$,

$$
C_{i}=\left[\begin{array}{lllll}
c_{1}^{i T} & c_{2}^{i T} & \ldots & c_{p-1}^{i T} & c_{p}^{T}
\end{array}\right]^{T}, \quad c_{j}^{i}= \begin{cases}0 & \text { if } i=j \\
c_{j} & \text { if } i \neq j\end{cases}
$$

Note that $c_{p}$ denotes a local signal and we will assume throughout that it is always available. In nominal conditions where all signals are available, $i=0$ and $C_{0}=\left[\begin{array}{lllll}c_{1}^{T} & c_{2}^{T} & \cdots & c_{p-1}^{T} & c_{p}^{T}\end{array}\right]^{T}=C$. Each of the measurements is the output of a sensor that can potentially fail, i.e. loss of the second sensor is represented as $i=2$ with $C_{2}=\left[\begin{array}{lllll}c_{1}^{T} & 0 & \cdots & c_{p-1}^{T} & c_{p}^{T}\end{array}\right]^{T}$. One remote signal loss at a time is considered for simplicity here but can be generalized for more than one.

\subsection{Conventional Control}

A conventional controller (CC) is designed to satisfy a desired level of dynamic performance when both the local and the remote signals are available. The performance of this controller, however, can deteriorate significantly following sudden loss of the remote signals. A regional pole-placement approach using LMIs is adopted for the design of CC. Unlike classical pole-placement approaches, such as tuning of lead-lag blocks or state feedback, the LMI approach allows the imposition of additional constraints.

The same methodology is subsequently used for the FTC design to ensure a fair comparison between FTC and CC. For the CC, the design formulation is linear and simpler as described next.

The controller is represented in state space form as

$$
\dot{x}_{c}(t)=A_{c} x_{c}(t)+B_{c} y(t), \quad u(t)=C_{c} x_{c}(t), \quad K_{c}(s) \stackrel{s}{=}\left[\begin{array}{c|c}
A_{c} & B_{c} \\
\hline C_{c} & 0
\end{array}\right]
$$

with $A_{c} \in \Re^{n \times n}, B_{c} \in \Re^{n \times p}$ and $C_{c} \in \Re^{q \times n}$. The closed-loop state dynamics of this controller is described as $\dot{\tilde{x}}=\tilde{A}_{i} \tilde{x}$ where

$$
\tilde{A}_{i}=\left[\begin{array}{cc}
A & B C_{c} \\
B_{c} C_{i} & A_{c}
\end{array}\right] .
$$


In this section we will design a controller for the nominal, fault free condition $(i=0)$. The control objective for the $\mathrm{CC}$ is to place the eigenvalues of (4) within a desired region of the complex plane. The following theorem [27] describes the solution approach.

\section{Theorem 1}

Let $i=0$. Then $\tilde{A}_{i}$ is stable and all its eigenvalues lie within the conic sector of the complex plane, shown in Fig. 2, if and only if there exists $\tilde{P}=\tilde{P}^{T}$ such that

$$
\tilde{P}>0, \quad\left[\begin{array}{cc}
\sin \theta_{i}\left(\tilde{A}_{i}^{T} \tilde{P}+\tilde{P} \tilde{A}_{i}\right) & \cos \theta_{i}\left(\tilde{A}_{i}^{T} \tilde{P}-\tilde{P} \tilde{A}_{i}\right) \\
\cos \theta_{i}\left(\tilde{P} \tilde{A}_{i}-\tilde{A}_{i}^{T} \tilde{P}\right) & \sin \theta_{i}\left(\tilde{A}_{i}^{T} \tilde{P}+\tilde{P} \tilde{A}_{i}\right)
\end{array}\right]<0,
$$

where $\theta_{i}$ is the inner angle of the cone shown in Fig. 2.

The formulation of this problem is bilinear but the nonlinearities can be eliminated by some appropriate change of controller variables [27]. These changes are implicitly defined in terms of the partition of the Lyapunov matrix $\tilde{P}$ and its inverse

$$
\tilde{P}=\left[\begin{array}{cc}
X & U \\
U^{T} & X_{c}
\end{array}\right], \quad \tilde{P}^{-1}=\left[\begin{array}{cc}
Y & V \\
V^{T} & Y_{c}
\end{array}\right],
$$

with $X, Y, U$ and $V \in \Re^{n \times n}$. Since $\tilde{P} \tilde{P}^{-1}=I$,

$$
U V^{T}=I-X Y
$$

It can been verified that $\tilde{P}$ satisfies the identity $\tilde{P} \Pi_{2}=\Pi_{1}$ where

$$
\Pi_{1}=\left[\begin{array}{cc}
X & I \\
U^{T} & 0
\end{array}\right], \quad \Pi_{2}=\left[\begin{array}{cc}
I & Y \\
0 & V^{T}
\end{array}\right] .
$$

Post- and pre-multiplying the first and second inequalities in (5) by the matrices

$$
\Pi_{2},\left[\begin{array}{cc}
\Pi_{2} & 0 \\
0 & \Pi_{2}
\end{array}\right],
$$

and their transposes, respectively, and introducing the following change of variables [27]

$$
\begin{aligned}
& \hat{C}_{c}=C_{c} V^{T}, \quad \hat{B}_{c}=U B_{c}, \\
& \hat{A}_{c}=X A Y+X B \hat{C}_{c}+U A_{c} V^{T}+\hat{B}_{c} C Y,
\end{aligned}
$$

the inequalities in (5) reduce to the following LMIs

$$
\begin{gathered}
{\left[\begin{array}{cc}
X & I \\
\star & Y
\end{array}\right]>0} \\
{\left[\begin{array}{cc}
\sin \theta_{0} L_{11} & \cos \theta_{0} L_{12} \\
\star & \sin \theta_{0} L_{11}
\end{array}\right]<0,}
\end{gathered}
$$

where $\star$ denotes a symmetric element in a symmetric matrix and where

$$
\begin{aligned}
& L_{11}=\left[\begin{array}{cc}
X A+A^{T} X+\hat{B}_{c} C+C^{T} \hat{B}_{c}^{T} & A^{T}+\hat{A}_{c} \\
\star & A Y+Y A^{T}+B \hat{C}_{c}+\hat{C}_{c}^{T} B^{T}
\end{array}\right], \\
& L_{12}=\left[\begin{array}{cc}
A^{T} X-X A+C^{T} \hat{B}_{c}^{T}-\hat{B}_{c} C & A^{T}-\hat{A}_{c} \\
\star & Y A^{T}-A Y+\hat{C}_{c}^{T} B^{T}-B \hat{C}_{c}
\end{array}\right] .
\end{aligned}
$$

The solution to these LMIs, together with (6), (8) and (9), can be used to calculate the controller realisation in (3). Note that one of $U$ and $V$ in (6) can be arbitrarily assigned, provided it is nonsingular. 


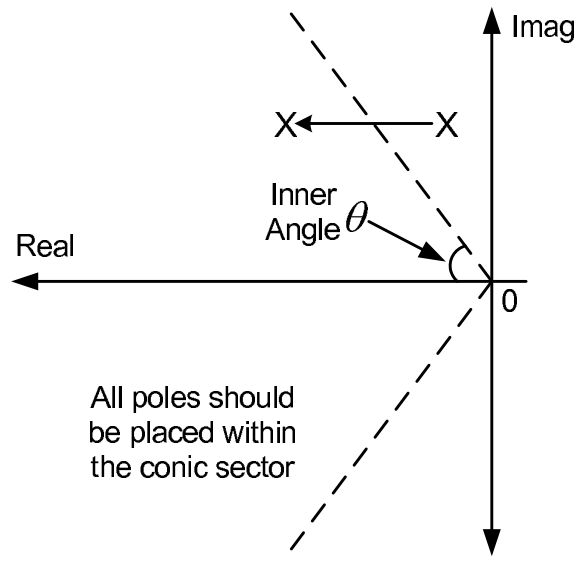

Figure 2. Conic sector with inner angle $\theta$ where all poles should be placed

\section{FAULT-TOLERANT CONTROL}

An FTC is required to not only achieve a desired level of dynamic performance when both local and remote signals are available but also guarantee a minimum level of performance following sudden loss of remote signals. In this section we present two approaches for FTC design: a passive FTC presented in Section 3.1 and a special type of active FTC presented in Section 3.2.

\subsection{Iterative procedure for passive FTC}

The problem of passive FTC $\left(\mathrm{FTC}_{\mathrm{p}}\right)$ design consists of synthesizing a single controller, if such a controller exists, that satisfies the design specifications for the family of plants as described in (2). The state space representation of the $\mathrm{FTC}_{\mathrm{p}}$ and its closed-loop state dynamics matrix are given as in (3) and (4) for $i=0,1, \ldots, p$. This controller is designed for the family of plants (2) such that:

$$
u(s)=K_{c}(s) y_{0}(s), \cdots, u(s)=K_{c}(s) y_{p}(s) .
$$

The requirement is that the eigenvalues of $\tilde{A}_{i}$ for $i=0,1, \ldots, p$ lie in the conic region described in Fig. 2. The constraints are the same as in (5), where in this case, $\theta_{i}$ is the required inner angle for the $i$ th system.

A linear non-iterative algorithm to design decentralized controllers for polytopic systems was presented in [28] and implemented in [29] for FTC design. The algorithms presented have the advantage of being linear and easy to implement, although they have a potential drawback: the value of the matrix $C_{c}$ in the controller is calculated and fixed to the value of $\hat{C}_{c} Y^{-1}$ before calculating the other control matrices. Consequently, the values of $A_{c}$ and $B_{c}$ depend on $C_{c}$. As a result, it may be possible that there is no solution for a specific value of $C_{c}$ but one may exist for other values. An iterative procedure can avoid this problem and can potentially improve the damping of the system.

Applying the same transformations to (5) as in CC, it turns out that it is not possible to perform the same change of variables as in (9) to linearize the inequalities. This is due to the problem of having multiple systems corresponding to the different possible fault scenarios. In order to linearize the bilinearities, applying the transformations (7) to (5) as before, we introduce again the following change of variables

$$
\hat{C}_{c}=C_{c} V^{T}, \quad \hat{B}_{c}=U B_{c},
$$

and in contrast to [27], we define $\hat{A}_{c}$ as

$$
\hat{A}_{c}=X A Y+X B \hat{C}_{c}+U A_{c} V^{T} .
$$


At this point, the inequalities in (5) reduce to the LMI in (10) and the inequality

$$
\left[\begin{array}{cc}
\sin \theta_{i} L_{11}^{i} & \cos \theta_{i} L_{12}^{i} \\
\star & \sin \theta_{i} L_{11}^{i}
\end{array}\right]<0
$$

for all $i$, where

$$
\begin{aligned}
L_{11}^{i}= & {\left[\begin{array}{cc}
X A+A^{T} X+\hat{B}_{c} C_{i}+C_{i}^{T} \hat{B}_{c}^{T} & A^{T}+\hat{A}_{c}+\hat{B}_{c} C_{i} Y \\
\star & A Y+Y A^{T}+B \hat{C}_{c}+\hat{C}_{c}^{T} B^{T}
\end{array}\right], } \\
L_{12}^{i}= & {\left[\begin{array}{cc}
A^{T} X-X A+C_{i}^{T} \hat{B}_{c}^{T}-\hat{B}_{c} C_{i} & A^{T}-\hat{A}_{c}-\hat{B}_{c} C_{i} Y \\
\star & Y A^{T}-A Y+\hat{C}_{c}^{T} B^{T}-B \hat{C}_{c}
\end{array}\right] }
\end{aligned}
$$

Note that the inequality in (13) is nonlinear due to the term $\hat{B}_{c} C_{i} Y$ in the off-diagonal blocks of (14) and (15). However, since this term is bilinear, there are many excellent methods for dealing with this case [30]. Since the open-loop is stable, it is possible to alternately fix the value of one of the two variables $\hat{B}_{c}$ or $Y$ to improve the damping in an iterative algorithm. Let $k$ denote the iteration number and note that, for a variable $Z$, we use the notation $Z(k)$ to denote its value at iteration $k$.

\section{Iterative Algorithm}

(i) Set $k=1$ and $\hat{B}_{c}(k)=0$, so that (13) is linear and feasible since the open-loop is stable.

(ii) Find the minimum value of $\theta_{i}(k)$ (reduce the angle of the conic sector to shift the modes to the left hand side of the complex plain) for which there exist a feasible solution to (10) and (13). Calculate $Y(k), X(k), \hat{C}_{c}(k), \hat{A}_{c}(k)$ and set $Y(k+1)=Y(k)$.

(iii) Set $k=k+1$

(iv) Find the minimum value of $\theta_{i}(k)$ (reduce the angle of the conic sector to shift the modes to the left hand side of the complex plain) for which there exist a feasible solution to (10) and (13). Calculate $\hat{B}_{c}(k), X(k), \hat{C}_{c}(k), \hat{A}_{c}(k)$ and set $\hat{B}_{c}(k+1)=\hat{B}_{c}(k)$.

(v) Set $k=k+1$ and go to (ii).

Note that $\theta_{i}(k+1) \leq \theta_{i}(k)$. The iterative procedure terminates when

$$
\theta_{i}(k)-\theta_{i}(k+1) \leq \epsilon
$$

where $\epsilon$ can be adjusted to a small value i.e. $10^{-3}$. When the iterative algorithm has converged, (16) is satisfied; to retrieve the elements of the $\operatorname{FTC}_{\mathrm{p}}\left(A_{c}, B_{c}\right.$ and $\left.C_{c}\right)$ use (11) and (12). Since we have used a linear approximation for the bilinear inequalities, there is no guarantee that the solution will converge to the optimal solution. However, in our experience, it does give improvement to the damping ratio as illustrated in the examples in Sections 6 and 7.

\subsection{Minimal switching FTC}

The conventional controller developed in Section 2.1 has the advantage that it is optimal for the fault free situation, although it lacks any guaranteed fault tolerance. On the other hand, the controller developed in Section 3.1 has the advantage that it is passive (resulting in ease of implementation) and fault-tolerant, although, since it requires the solution of bilinear matrix inequalities, evaluating the optimal solution is difficult and we will generally have to settle for suboptimal approximations.

In the case that we have full information about the faults, it is possible to design several controllers, one for each fault scenario, and then use the fault information to switch between these controllers. This has the advantage that the controller will combine fault tolerance with good performance, however, this drastically complicates the control implementation. As an alternative to both passive and full switching control, in this section we propose what we call a minimal switching FTC procedure in which one controller is designed, but where a simple switch is incorporated into the controller, thus combining the advantages of both passive and active FTC schemes.

We consider switching control of the form

$$
K_{c}^{i}(s) \stackrel{s}{=}\left[\begin{array}{c|c}
A_{c}^{i} & B_{c} \\
\hline C_{c} & 0
\end{array}\right] .
$$




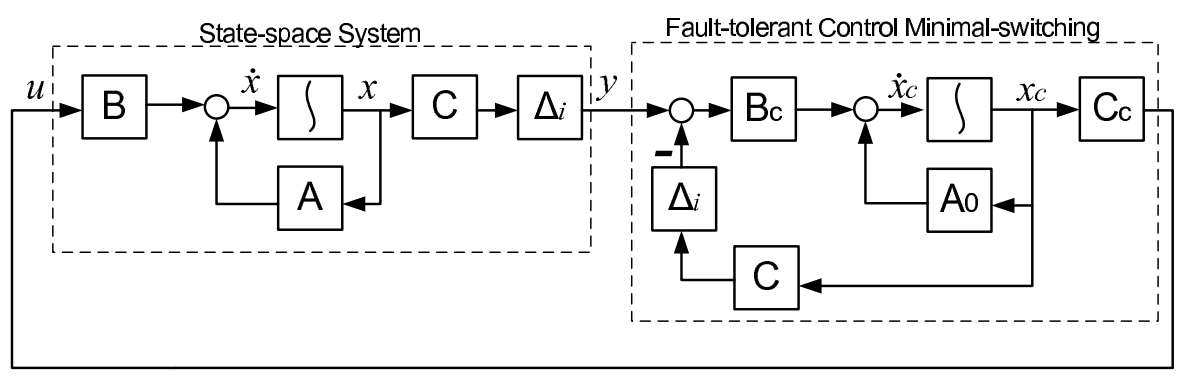

Figure 3. Minimal switching scheme.

Applying the transformations (7) to (5) as before, and introducing the following change of variables

$$
\hat{C}_{c}=C_{c} V^{T}, \quad \hat{B}_{c}=U B_{c}, \quad \hat{A}_{c}=X A Y+X B \hat{C}_{c}+U A_{c}^{i} V^{T}+\hat{B}_{c} C_{i} Y,
$$

the problem can be expressed as the solution of the LMIs (10) and (13) for all $i$, where

$$
\begin{aligned}
L_{11}^{i}= & {\left[\begin{array}{cc}
X A+A^{T} X+\hat{B}_{c} C_{i}+C_{i}^{T} \hat{B}_{c}^{T} & A^{T}+\hat{A}_{c} \\
\star & A Y+Y A^{T}+B \hat{C}_{c}+\hat{C}_{c}^{T} B^{T}
\end{array}\right], } \\
L_{12}^{i}= & {\left[\begin{array}{cc}
A^{T} X-X A+C_{i}^{T} \hat{B}_{c}^{T}-\hat{B}_{c} C_{i} & A^{T}-\hat{A}_{c} \\
\star & Y A^{T}-A Y+\hat{C}_{c}^{T} B^{T}-B \hat{C}_{c}
\end{array}\right] }
\end{aligned}
$$

Note that this formulation is now linear. Next, we give an interpretation of the definition for $\hat{A}_{c}$ in (17). Let $V=Y$ (recall that one of $V$ and $U$ are free). Solving (17) for $A_{c}^{i}$ gives

$$
A_{c}^{i}=U^{-1} \hat{A}_{c} Y^{-1}-U^{-1} X A-U^{-1} X B C_{c}-B_{c} C_{i}
$$

Defining $A_{0}=U^{-1} \hat{A}_{c} Y^{-1}-U^{-1} X A-U^{-1} X B C_{c}$ and letting $C_{i}=\Delta_{i} C$, where the diagonal matrix $\Delta_{i}$ represents a switching matrix with a zero in the $i$ th diagonal entry representing a fault and a 1 representing no fault in the $i$ th sensor, we can write

$$
A_{c}^{i}=A_{0}-B_{c} \Delta_{i} C
$$

The implementation of the proposed FTC architecture is shown in Figure 3. Notice that the parameters of the controller $A_{0}, B_{c}$ and $C_{c}$ are time-invariant. The time variation is restricted to the switching in $\Delta_{i}$, hence our term minimal switching controller, which we refer to as $\mathrm{FTC}_{m s}$.

\section{TEST SYSTEM}

A nonlinear equivalent of the Nordic system is used for the case study. The detailed model with approximately 3000 buses, 4000 branches and 1100 generators [31,25] was reduced down to a 20 generator, 36 bus equivalent system shown in Fig. 4. The aim was to retain the modal behavior of the two most critical poorly damped inter-area modes. A static VAr compensator (SVC) is included in the model at Hasle substation (5101) in south-east Norway in the same way it exists in practice. The nonlinear reduced equivalent is then linearized around an operating point; the total number of state variables for the linearized version of the equivalent system is 296 .

There are two critical inter-area modes, 0.29 and $0.55 \mathrm{~Hz}$ with $4.8 \%$ and $5.4 \%$ damping, respectively, under nominal condition; see Table I. The first mode $(0.29 \mathrm{~Hz})$ comprises the Finnish generators swinging against the rest whereas the second mode $(0.55 \mathrm{~Hz})$ involves the generators in the north of Finland, Sweden and Norway swinging against those in the southern parts of these countries. 


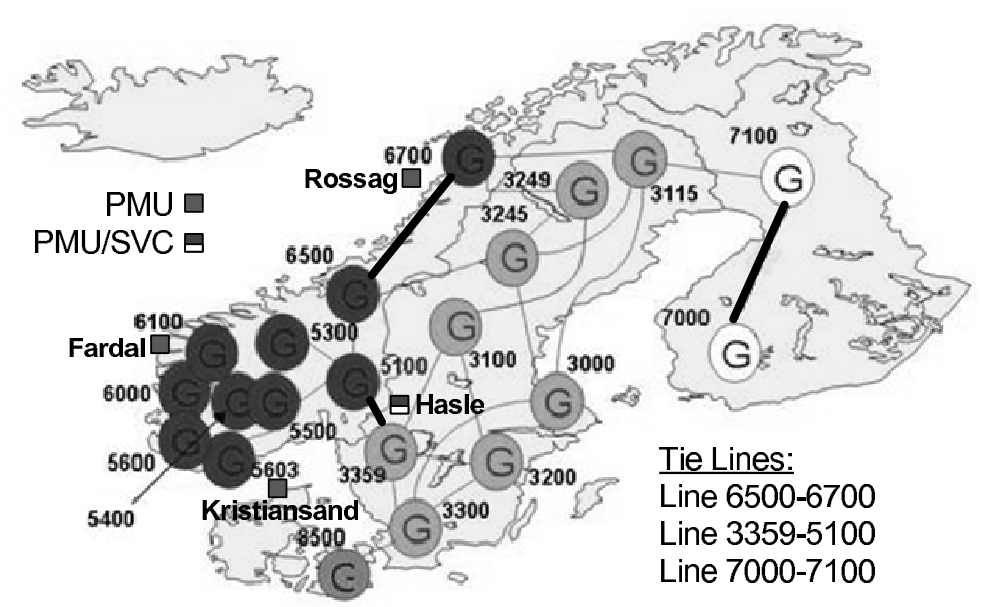

Figure 4. Nordic equivalent system where the location of the PMUs and key tie-lines are marked.

Following critical contingencies such as outage of one of the parallel lines connecting (i) 6500-6700 (Norwegian coast line), (ii) 7100-7000 (Finnish line) or (iii) 3359-5101 (Hasle line), the damping of the inter-area modes could be as low as $2-3 \%$ requiring improvement through supplementary control. The challenge is to ensure satisfactory damping of both modes by designing a POD for the SVC at Hasle.

With a number of phasor measurement units (PMUs) (only four in Norway and two in Finland are considered for this study) installed throughout the Nordic system, remote feedback signals were also considered in addition to the local signals. The difference between voltage angles available from the PMUs were chosen as potential candidates [31] for remote signals.

The voltage angle difference between the PMUs at 6100 (at Nedre Rossaga) and 7000 (in southern Finland) has high residue magnitude for both modes and the phase angles are also in the same direction (see the Appendix for details of the procedure used for signal selection). Thus, based on both the magnitude and phase angle of the residues [32], 6100-7000 was selected as the most appropriate signal for the POD, highlighted in Table II.

Out of the available local signals, the magnitude and phase angle of the line currents were found to be the most effective candidates. The phase angle of the current in line 5101-5501 was selected as the most appropriate local signal based on both the magnitude and phase angle of the residue.

\section{CONTROL DESIGN}

The control design formulations presented in Sections 2.1, 3.1 and 3.2 result in controllers that have the same order as the plant, which is too high for feasible control synthesis. To get around this problem, the linearized model of the test system (described in Section 4) with 296 states was first reduced to a 12th order equivalent using balanced stochastic model truncation through the Schur model reduction method [33]. The similarity between the frequency responses of the original and reduced systems were used to validate the accuracy of the reduced order model.

One case study, using the nonlinear equivalent system, comparing the performance of three different controllers (each of order 12) using local and remote signals: conventional (CC), passive $\left(\mathrm{FTC}_{\mathrm{p}}\right)$ and minimal switching $\left(\mathrm{FTC}_{\mathrm{ms}}\right)$, is presented in Section 7 below. The controllers were designed using the approaches described in Sections 2.1, 3.1 and 3.2 respectively. Similar to CC, the FTCs improve the damping of the two critical modes up to $10 \%$ under normal conditions. In addition, the FTCs maintain the damping above $8 \%$ even with the loss of the remote signal. However, with CC the damping of mode 1 reduces to $4.9 \%$ in absence of the remote signal. 
(a)

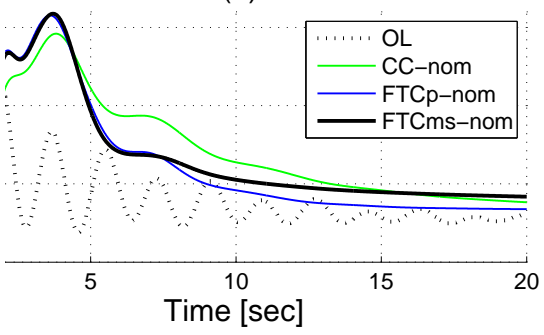

Nominal Conditions

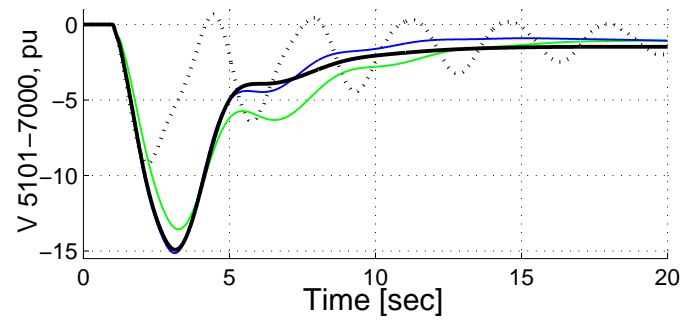

(d)
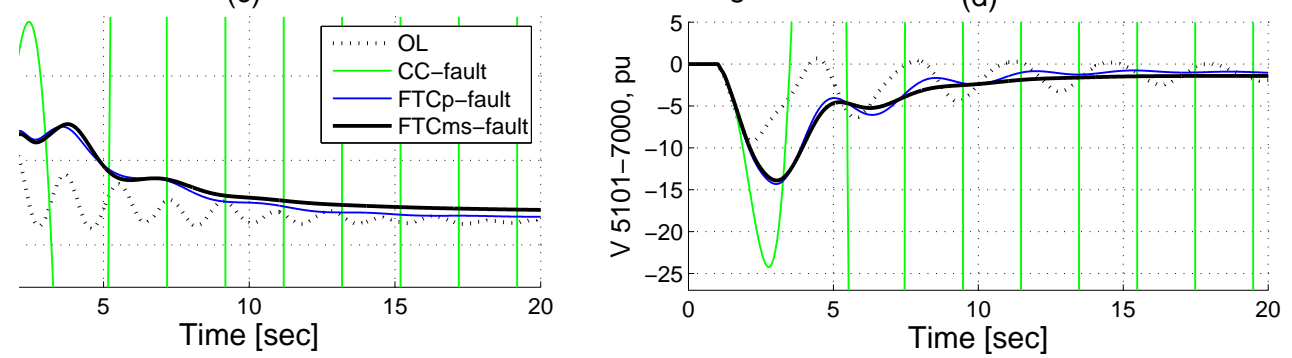

Figure 5. Comparison of dynamic performance of the controllers under normal and loss of one remote signal condition against the open-loop (OL) response

But first, one case study using the reduced linear model (of order 12) is presented to illustrate how the stability of the closed-loop system could be affected following the loss of one of the remote signals when this problem is not considered $a$ priori.

\section{CONTROL PERFORMANCE: LINEAR SIMULATIONS}

In this section, the dynamic performance of the conventional controller (CC), the passive FTC $\left(\mathrm{FTC}_{\mathrm{p}}\right)$ and the minimal switching $\mathrm{FTC}\left(\mathrm{FTC}_{\mathrm{ms}}\right)$ are compared both in normal and 'fault' (when one remote signal is lost) conditions. The simulations and the control design are based on the linear 12 state model. All controllers were designed using the same local (phase angle of current in line 5101-5501) and two remote feedback signals (voltage angle differences 6100-7000 and 5101-7000) based on resides magnitudes as described in Tables II and III, respectively. The objective is to illustrate that stability of the closed-loop system can be affected when using the CC if more than one remote signals are used and one of them is lost.

Figure 5 shows the dynamic performance of the closed-loop between the single-input, threeoutputs, 12 state Nordic power grid and the three-input, single-output, 12-state controllers CC, $\mathrm{FTC}_{\mathrm{p}}$ and $\mathrm{FTC}_{\mathrm{ms}}$ in the linear simulation. Here, it can be seen that in nominal conditions all controllers provide satisfactory performance and fast settling times (Fig. 5 (a) and (b)) to eliminate the oscillations (dotted trace) caused by a small disturbance at the input of the system. However, after the loss of one of the remote signals (voltage angle differences 6100-7000), the dynamic response using the $\mathrm{CC}$ is unstable (green $\backslash$ solid-light trace in Fig. 5 (c) and (d)) while the dynamic responses of $\mathrm{FTC}_{\mathrm{p}}$ and $\mathrm{FTC}_{\mathrm{ms}}$ maintain performance levels which are comparable to the case of nominal conditions (blue $\backslash$ solid-medium and black $\backslash$ solid-thick trace in Fig. 5 (c) and (d)). Note that the plots in Fig. 5 (c) and (d) have been "chopped off" to enable the comparison between the controllers in this particular faulty situation. 
(a)
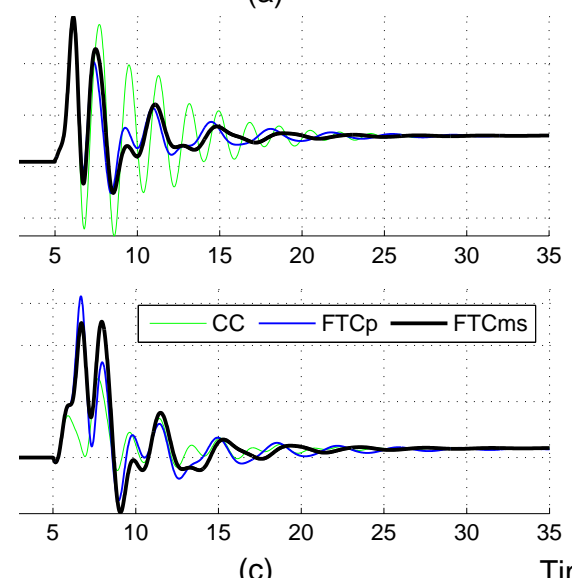

(c)
Nominal Conditions
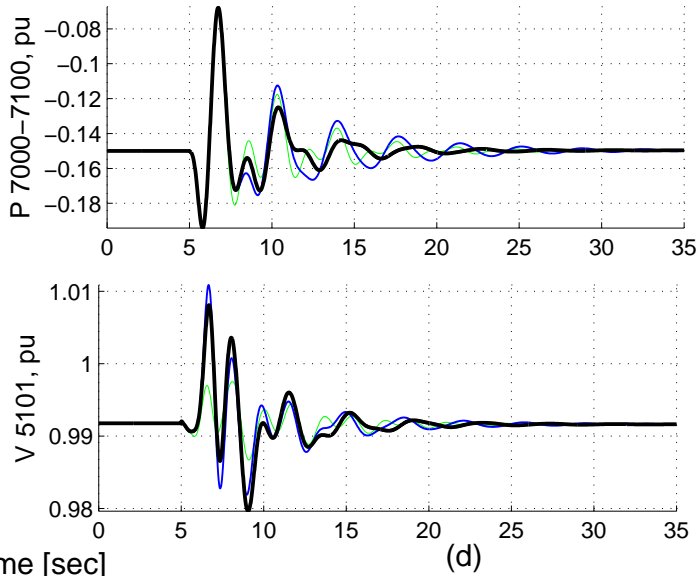

Figure 6. Comparison of dynamic performance with $\mathrm{CC}, \mathrm{FTC}_{p}$ and $\mathrm{FTC}_{m s}$ under normal (remote signal present) condition

\section{CONTROL PERFORMANCE: NONLINEAR SIMULATIONS}

Since our controllers were designed based on a reduced-order linearized plant model, it is therefore essential that the performance of the controller should be validated using the actual nonlinear model of the system. In this section the dynamic performance of the fault-tolerant controllers $\left(\mathrm{FTC}_{\mathrm{p}}\right.$ and $\mathrm{FTC}_{\mathrm{ms}}$ ) is compared against a conventional controller (CC) under both normal and fault conditions. All controllers were designed using the same local and remote feedback signals mentioned in Section 4. The design specification in all cases was to achieve at least $10 \%$ damping. However, for $\mathrm{FTC}_{\mathrm{p}}$ and $\mathrm{FTC}_{\mathrm{ms}}$, the target damping was reduced to $8 \%$ for the fault condition to obtain a feasible solution. A short circuit followed by outage of tie-line 6500-6700 was simulated to compare the dynamic performance.

\subsection{Normal Condition}

Under normal condition, the dynamic performance of $\mathrm{CC}$ as seen from the power flow through the lines 3359-5101 (Fig. 6 (a)) and 7000-7100 (Fig. 6 (b)) is acceptable, while the dynamic response of both fault-tolerant controllers as seen from the power flow through lines 3359-5101 (Fig. 6 (a)) is slightly less oscillatory. Note that similar control effort is required by both FTCs as indicated in $B_{S V C}$ (Fig. 6 (c)) and also the bus voltage V 5101 (Fig. 6 (d)).

\subsection{Loss of Remote Signals}

The performance of the controllers is compared in Fig. 7 in the face of loss of the remote signal. The variation of the power flow through line 3359-5101 (Fig. 7 (a)) is not affected much by the loss of the remote signal for any controller. As mentioned before, this power flow predominantly reflects mode 2 which has adequate observability (residue) in the local signal which explains the above trend. On the other hand, power flow through 7000-7100 has prominent presence of mode 1 which is not adequately observable in the local signal. Hence, with $\mathrm{CC}$, loss of the remote signal results in significant deterioration in the oscillatory behavior of this signal (Fig. 7 (b)). However, with both FTCs, satisfactory performance is maintained for both power flows with only the local signal, with $\mathrm{FTC}_{m s}$ exhibiting a slightly less oscillatory response than $\mathrm{FTC}_{p}$. Similar to the normal condition, the control effort required by the FTCs is slightly higher than and $\mathrm{CC}$ as evident through the larger excursions on $B_{S V C}$ (Fig. 7 (c)). 
(a)
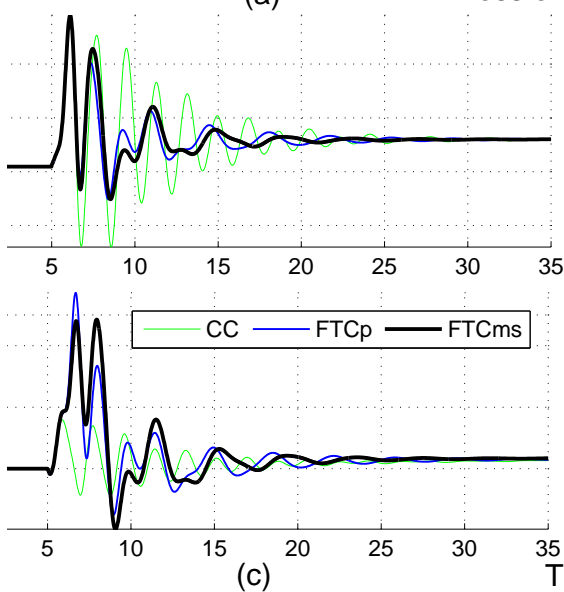

(c)
Loss of Remote Signal
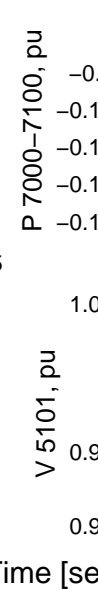

(b)

Figure 7. Comparison of dynamic performance with $\mathrm{CC}, \mathrm{FTC}_{p}$ and $\mathrm{FTC}_{m s}$ following loss of remote signal

Although both FTCs require larger control effort than CC to achieve a desired damping under normal condition, the former can guarantee an acceptable performance level when the remote signal is lost unlike the $\mathrm{CC}$ which leads to significant deterioration in dynamic behavior.

\section{CONCLUSION}

In this paper passive and minimal switching fault-tolerant control design methodologies are proposed to enhance the damping of inter-area oscillations in power grids. Two case studies, one in a linear simulation and the second in a nonlinear simulation, in the Nordic equivalent system show that the closed-loop response could deteriorate or even become unstable if the remote signals are lost. Under normal condition (when both local and remote signals are present) the fault-tolerant controllers require more control effort as compared to a conventional controller in order to achieve similar performance. However, our case studies confirm that the proposed fault-tolerant controllers are able to guarantee stability and produce acceptable performance in case of loss of the remote signals, while the response using the conventional controller is unacceptable if a fault occurs.

\section{APPENDIX}

This section presents the procedure applied for the selection of the feedback signals.

\section{Residues and Signal Selection}

Consider the linear system in (1), where the eigenvalues are defined as $\lambda_{i}=\alpha_{i} \pm j \omega_{i}$. The modal controllability index

$$
M C_{i j}=v_{i} B_{j}
$$

indicates the controllability of the $i$ th mode using the $j$ th input where $v_{i}$ is the left eigenvector of the system (1) for the corresponding mode $\lambda_{i}$. The modal observability index

$$
M O_{i k}=C_{k} w_{i}
$$

indicates the observability of the $i$ th mode of the $k$ th output, where $w_{i}$ is the right eigenvector of the system (1) for the corresponding mode $\lambda_{i}$. Thus, the signal having highest observability index is chosen as the feedback signal. The higher the value of these indices, the better the effectiveness of 
the controller. These measures are normally expressed in the form of residues $R_{i}=\left(M C_{i j}\right)\left(M O_{i k}\right)$ and can be written as

$$
R_{i}=\left|R_{i}\right| \angle \delta_{i}
$$

where $\left|R_{i}\right|$ and $\delta_{i}$ are the absolute value and the angle of the residue, respectively, for a particular $i$ th mode. A high value $\left|R_{i}\right|$ is essential for an effective controller. The angle $\delta_{i}$ is normally used to calculate phase compensation; more details can be found in [34].

\section{ACKNOWLEDGEMENT}

Funding from ABB, Switzerland (Grant EESC P26939) and technical input from staff within ABB are gratefully acknowledged.

\section{REFERENCES}

1. Li X, Liu HHT. A passive fault tolerant flight control for maximum allowable vertical tail damaged aircraft. Journal of Dynamic Systems, Measurement, and Control 2012; 134(3):031006.

2. Sloth C, Esbensen T, Stoustrup J. Active and passive fault-tolerant lpv control of wind turbines. American Control Conference (ACC), 2010, 2010; 4640 -4646.

3. Lianqing S, Xiaodan Z, Jiqing Q, Changjie Z, Yanchun Z, Yuming G. Robust passive fault-tolerant control for uncertain non-linear stochastic systems with distributed delays. Control Conference (CCC), 2010 29th Chinese, 2010; $1949-1953$.

4. Yang Y, Wang YJ, Qiu JQ, Niu YR. Robust Hinf; passive fault-tolerant control for uncertain singular systems. Machine Learning and Cybernetics (ICMLC), 2011 International Conference on, vol. 3, 2011; 1308 -1312.

5. Gao Z, Jiang B, Shi P, Liu J, Xu Y. Passive fault-tolerant control design for near-space hypersonic vehicle dynamical system. Circuits, Systems, and Signal Processing 2012; 31:565-581.

6. Veillette R, Medanic J, Perkins W. Design of reliable control systems. Automatic Control, IEEE Transactions on mar 1992; 37(3):290 -304.

7. Vidyasagar M, Viswanadham N. Reliable stabilization using a multi-controller configuration. Automatica 1985; 21(5):599-602.

8. Liang YW, Liaw DC, Lee TC. Reliable control of nonlinear systems. Automatic Control, IEEE Transactions on apr 2000; 45(4):706 -710.

9. Liang YW, Xu SD, Tsai CL. Study of vsc reliable designs with application to spacecraft attitude stabilization. Control Systems Technology, IEEE Transactions on march 2007; 15(2):332 -338.

10. Liao F, Wang JL, Yang GH. Reliable robust flight tracking control: an lmi approach. Control Systems Technology, IEEE Transactions on jan 2002; 10(1):76-89.

11. Huang D, Nguang SK. Robust fault estimator design for uncertain networked control systems with random time delays: An ilmi approach. Information Sciences 2010; 180(3):465 - 480.

12. Lien CH, Yu KW, Lin YF, Chung YJ, Chung LY. Robust reliable h8 control for uncertain nonlinear systems via $1 \mathrm{mi}$ approach. Applied Mathematics and Computation 2008; 198(1):453 - 462.

13. Niemann H, Stoustrup J. An architecture for fault tolerant controllers. International Journal of Control 2005; 78(14):1091-1110.

14. Niemann H, Stoustrup J. Passive fault tolerant control of a double inverted penduluma case study. Control Engineering Practice 2005; 13(8): 1047 - 1059.

15. Stoustrup J, Niemann H. Fault tolerant control for unstable systems: a linear time varying approach. American Control Conference, 2004. Proceedings of the 2004, vol. 2, 2004; $1794-1798$ vol.2.

16. Makarov Y, Reshetov V, Stroev A, Voropai I. Blackout prevention in the united states, europe, and russia. Proceedings of the IEEE nov 2005; 93(11):1942 -1955, doi:10.1109/JPROC.2005.857486.

17. Kamwa I, Heniche A, Trudel G, Dobrescu M, Grondin R, Lefebvre D. Assessing the technical value of FACTSbased wide-area damping control loops. proceedings of IEEE Power Engineering Society General Meeting, 2005, 2005; 1734-1743 Vol. 2.

18. Chakrabortty A. Wide-area damping control of power systems using dynamic clustering and tcsc-based redesigns. Smart Grid, IEEE Transactions on sept 2012; 3(3):1503 -1514, doi:10.1109/TSG.2012.2197029.

19. Kamwa I, Grondin R, Hebert Y. Wide-area measurement based stabilizing control of large power systemsa decentralized/hierarchical approach. Power Systems, IEEE Transactions on feb 2001; 16(1):136 -153, doi: $10.1109 / 59.910791$.

20. Dotta D, e Silva AS, Decker IC. Wide-area measurements-based two-level control design considering signal transmission delay. IEEE Transactions on Power Systems 2009; 24(1):208-216.

21. Chaudhuri B, Majumder R, Pal B. Wide-area measurement-based stabilizing control of power system considering signal transmission delay. IEEE Transactions on Power Systems 2004; 19(4):1971-1979.

22. Chaudhuri NR, Ray S, Majumder R, Chaudhuri B. A new approach to continuous latency compensation with adaptive phasor power oscillation damping controller (pod). IEEE Transactions on Power Systems 2010; 25(2):939946.

23. Chaudhuri NR, Chakraborty D, Chaudhuri B. An architecture for FACTS controllers to deal with bandwidthconstrained communication. IEEE Transactions on Power Delivery 2011; 26(1):188-196. 
24. Mekki K, Snyder A, HadjSaid N, Feuillet R, Georges D, Margotin T. Damping controller input-signal loss effects on the wide-area stability of an interconnected power system. IEEE Power Engineering Society Summer Meeting, vol. 2, 2000; 1015-1019.

25. Chaudhuri N, Domahidi A, Majumder R, Chaudhuri B, Korba P, Ray S, Uhlen K. Wide-area power oscillation damping control in nordic equivalent system. Generation, Transmission Distribution, IET october 2010; 4(10):1139 -1150 , doi:10.1049/iet-gtd.2009.0478.

26. Hingorani NG, Gyugyi L. Understanding FACTS: concepts and technology of flexible AC transmission systems. IEEE Press: New York, 2000.

27. Chilali M, Gahinet P. H-infinity design with pole placement constraints: an 1mi approach. Automatic Control, IEEE Transactions on mar 1996; 41(3):358 -367, doi:10.1109/9.486637.

28. Ramos R, Alberto L, Bretas N. A new methodology for the coordinated design of robust decentralized power system damping controllers. Power Systems, IEEE Transactions on feb 2004; 19(1):444 - 454, doi:10.1109/TPWRS.2003. 820690.

29. Segundo Sevilla F, Jaimoukha I, Chaudhuri B, Korba P. Fault-tolerant wide-area control for power oscillation damping. IEEE Power and Energy Society General Meeting, 2012.

30. Tuan H, Apkarian P, Nakashima Y. A new lagrangian dual global optimization algorithm for solving bilinear matrix inequalities. Intternational Journal of Robust and Nonlinear Control 2000; 10:561-578.

31. Johansson E, Uhlen K, Leirbukt A, Korba P, Gjerde J, Vormedal L. Coordinating power oscillation damping control using wide area measurements. Power Systems Conference and Exposition, 2009. PSCE '09. IEEE/PES, 2009; 1 -8, doi:10.1109/PSCE.2009.4840162.

32. Ray S, Chaudhuri B, Majumder R. Appropriate signal selection for damping multi-modal oscillations using low order controllers. proceedings of IEEE Power Engineering Society General Meeting, 2008, Pittsburgh, 2008.

33. Safonov MG, Chiang RY. Model reduction for robust control: A schur relative-error method. American Control Conference, 1988, 1988; $1685-1690$.

34. Rogers G. Power systems oscillations. Kluwer Academic Publishers, 2000. 
Table I. Damping and Frequencies of the Inter-Area Modes under normal conditions

\begin{tabular}{|c|c|c|}
\hline \multicolumn{3}{|c|}{ Open-loop Nominal Conditions } \\
\hline Mode No. & $\begin{array}{c}\text { Frequency } \\
(\mathrm{Hz})\end{array}$ & $\begin{array}{c}\text { Damping Ratio } \zeta \\
(\%)\end{array}$ \\
\hline Mode 1 & 0.29 & 4.9 \\
Mode 2 & 0.55 & 5.4 \\
\hline
\end{tabular}

Table II. Remote signal selection: Magnitude and phase angle of residues for difference between relevant voltage angles

\begin{tabular}{|c|c|c|c|c|}
\hline & \multicolumn{2}{|c|}{ Mode 1 } & \multicolumn{2}{c|}{ Mode 2 } \\
\cline { 1 - 3 } \cline { 1 - 2 } Signal & Mag & Angle $($ deg) & Mag & Angle (deg) \\
\cline { 2 - 5 } $5101-5603$ & 0.03 & -64 & 0.68 & -80 \\
$5101-7000$ & 2.28 & 110 & 1.55 & 83 \\
$5101-7100$ & 1.26 & 107 & 1.97 & 94 \\
$5603-6700$ & 0.34 & 109 & 3.41 & 97 \\
$5603-7100$ & 1.29 & 107 & 2.65 & 96 \\
$\mathbf{6 1 0 0}-\mathbf{7 0 0 0}$ & $\mathbf{2 . 3 2}$ & $\mathbf{1 1 0}$ & $\mathbf{2 . 4 4}$ & $\mathbf{8 8}$ \\
$6700-7000$ & 1.97 & 111 & 1.26 & -67 \\
$7000-7100$ & 1.02 & -66 & 0.53 & 127 \\
\hline
\end{tabular}

Table III. Local Signals: Magnitude and phase angle of residues for Currents

\begin{tabular}{|c|c|c|c|c|}
\hline & \multicolumn{2}{|c|}{ Mode 1} & \multicolumn{2}{|c|}{ Mode 2} \\
\hline Signal & Mag & Angle (deg) & Mag & Angle (deg) \\
\hline$|I| 3359-5101$ & 0.28 & -62.09 & 4.84 & -81.44 \\
\hline$I_{\theta} 3359-5101$ & 0.35 & 100.43 & 0.35 & 44.44 \\
\hline$|I| 5101-5501$ & 0.21 & -64.84 & 3.76 & -82.20 \\
\hline$I_{\theta} \mathbf{5 1 0 1}-\mathbf{5 5 0 1}$ & 0.73 & 113 & 6.31 & 97.52 \\
\hline$|I| 5100-5100$ & 0.38 & -56.46 & 5.52 & -80.70 \\
\hline$I_{\theta} 5100-5100$ & 0.48 & 104.51 & 2.27 & 89.24 \\
\hline
\end{tabular}

